\title{
Earth microbial co-occurrence network reveals interconnection pattern across microbiomes
}

\author{
Bin Ma ${ }^{1,2}$, Yiling Wang ${ }^{1}$, Shudi Ye ${ }^{1}$, Shan Liu ${ }^{1}$, Erinne Stirling ${ }^{1,2}$, Jack A. Gilbert ${ }^{3}$, Karoline Faust ${ }^{4}$, \\ Rob Knight ${ }^{5}$, Janet K. Jansson ${ }^{6}$, Cesar Cardona ${ }^{7}$, Lisa Röttjers ${ }^{4}$ and Jianming $\mathrm{Xu}^{1,2^{*}}$ (D)
}

\begin{abstract}
Background: Microbial interactions shape the structure and function of microbial communities; microbial co-occurrence networks in specific environments have been widely developed to explore these complex systems, but their interconnection pattern across microbiomes in various environments at the global scale remains unexplored. Here, we have inferred an Earth microbial co-occurrence network from a communal catalog with 23,595 samples and 12,646 exact sequence variants from 14 environments in the Earth Microbiome Project dataset.
\end{abstract}

Results: This non-random scale-free Earth microbial co-occurrence network consisted of 8 taxonomy distinct modules linked with different environments, which featured environment specific microbial co-occurrence relationships. Different topological features of subnetworks inferred from datasets trimmed into uniform size indicate distinct co-occurrence patterns in the microbiomes of various environments. The high number of specialist edges highlights that environmental specific co-occurrence relationships are essential features across microbiomes. The microbiomes of various environments were clustered into two groups, which were mainly bridged by the microbiomes of plant and animal surface. Acidobacteria Gp2 and Nisaea were identified as hubs in most of subnetworks. Negative edges proportions ranged from $1.9 \%$ in the soil subnetwork to $48.9 \%$ the non-saline surface subnetwork, suggesting various environments experience distinct intensities of competition or niche differentiation. Conclusion: This investigation highlights the interconnection patterns across microbiomes in various environments and emphasizes the importance of understanding co-occurrence feature of microbiomes from a network perspective. Keywords: Co-occurrence patterns, Earth microbiomes, Genelist edges, Network hubs, Negative co-occurrence, Specialist edges, Microbial network topology

\section{Background}

Most microorganisms do not live in isolation; they thrive in communities with large numbers and develop close interactions that generate increased benefits for the group $[1,2]$. Microorganisms can establish a range of relationships including mutualism (such as antibiotic

\footnotetext{
*Correspondence: jmxu@zju.edu.cn

${ }^{1}$ Institute of Soil and Water Resources and Environmental Science, College of Environmental and Resource Sciences, Zhejiang University, Hangzhou, 310058, China

${ }^{2}$ Zhejiang Provincial Key Laboratory of Agricultural Resources and Environment, Hangzhou, 310058, China

Full list of author information is available at the end of the article
}

resistance conferral), commensalism (such as crossfeeding on compounds produced by other members), synergism (such as syntrophic cooperation), competition (such as niche exclusion), parasitism (such as infecting bacteria), predation (such as ciliates feeding on bacteria), antagosim (such as biocontrol agents), and amensalism (such as inducing a detrimental environment). These ecological interactions are critical evolutionary pressures for natural selection during microbial evolution. This premise is encapsulated by the Red Queen hypothesis, which emphasizes the coevolution of species, wherein established species evolve cooperatively through conditional 
dependencies [3]. Alternatively, the Black Queen hypothesis, which states that leaky metabolite production in certain species will lead to a reduction in corresponding functions in other species, provides an optional evolutionary possibility for the development of metabolic dependencies [4]. Adaptation in one species may increase selection pressure on another species, giving rise to antagonistic coevolution. Negative interactions (such as competition, parasitism, and predation) drive evolution by selective pressure [5], whereas positive interactions (such as mutualism) drive evolution by enhancing biological fitness [6]. Microbial interactions can partially explain genetic diversity in microbial populations. For example, certain functional genes can be dropped in a microbial genome due to random mutations and selective pressure if those functions are satisfied by other community members, which leads to low and medium gene frequencies, enabling ecological frequency-dependent selective pressure to drive microbial evolution [7]. Meanwhile, microbial interactions could also be reshaped by gaining adaptive genes to extend niche breadth, which alters interaction patterns [8].

Due to the poor mechanistic understanding of microbial community assembly, we found inconsistent prediction performance of microbial community structure in a wide range of fields [9-11]. Solving this unpredictability requires a comprehensive understanding of all aspects of microbiomes, including microbial interaction patterns [2]. Microbial co-occurrence networks are widely applied to explore connections in microbial communities. Nodes and edges in microbial co-occurrence network usually represent microbes and statistically significant associations between nodes, respectively. However, a systematic evaluation of microbial network inference as a tool for interaction prediction has highlighted this tool's low accuracy and the biological implications of network properties are unclear [12] . Nevertheless, modules in microbial co-occurrence networks may be indicative of ecological processes governing community structure, such as niche filtering and habitat preference [13]. Additionally, microbial co-occurrence network allows to predict hub species and potential species interactions [12]. Global microbial co-occurrence networks can provide a valuable resource for unravelling microbial co-occurrence patterns and their driving mechanisms. Chaffron et al. inferred a global network of co-existing microbes across environments from 298,591 16S rRNA sequences from the Greengenes database and found that phylogenetically close taxa coexisted more frequently [14]. Recent advances of highthroughput sequencing provide an opportunity for predicting microbial co-occurrence patterns from large-scale microbial community studies. For example, Lima-Mendez et al. inferred a global plankton co-occurrence network from the dataset of Tara Oceans, including 313 samples collected from 8 oceanic provinces. This network provides a resource for ocean microbial co-occurrence across several size fractions and depths and demonstrates the value of microbial co-occurrence networks for the formulation of ecological hypotheses such as differences in the role of top-down control across phytoplankton groups. Moreover, this network also helps to determine the role of global trends (generalist edges) and local signals (specialist edges) in driving entire plankton interactome [13]. The Earth Microbiome Project (EMP) is a public database and a framework for crowdsourced sample collection with standardized sequencing and metadata curation [15]. This database provides microbial community resources for cataloging global microbiota at an unprecedented scale for investigating large scale ecological patterns and exploring microbial community assembly theories. Here, we have inferred a global microbial co-occurrence network, describing microbial co-occurrence patterns using a dataset of 23,595 samples encompassing 14 environments from the EMP dataset. We used this network to explore the wired pattern among microbial communities in 14 environments.

\section{Results}

\section{Earth microbial co-occurrence network}

Fourteen microbial co-occurrence networks representing different environments were constructed, comprising 12,646 exact sequence variants (ESVs). To reduce noise and false-positive predictions, network inclusion was restricted to ESVs present in at least $10 \%$ of samples; we also used conservative statistical cut-off values (see "Materials and methods" section). The 14 networks were merged into a single Earth microbial co-occurrence network by overlapping the vertices and edges; the final network consists of 2928 vertices and 54,299 edges after removing unconnected vertices (Fig. 1a). The scale-free property $\left(R^{2}=0.19, P<0.001\right)$ and independency between abundance and degree $\left(R^{2}=-0.08, P=\right.$ $0.07)$ suggest a non-random co-occurrence pattern in this microbial network (Fig. S1). As ESVs were annotated to their representative microbial taxa, we were able to identify 812 taxa-pairs that were present more than twice in the global microbial co-occurrence network. We validated 432 co-occurrence edges, 15 intra-taxa edges, and 6 competition edges via literature mining (Data file S1). Although this only accounts for $1.5 \%$ of edges in the global microbial co-occurrence network, those 812 taxa pairs account for $30 \%$ of the edges presented in more than 6 environments.

This global network exhibits a high degree of modularity, but $87.9 \%$ of vertices were accounted for by only 8 of the 53 total modules (Fig. S2). Among these eight modules, the first 5 are densely nested into a giant module, while modules 6,7 , and 8 remain isolated from 


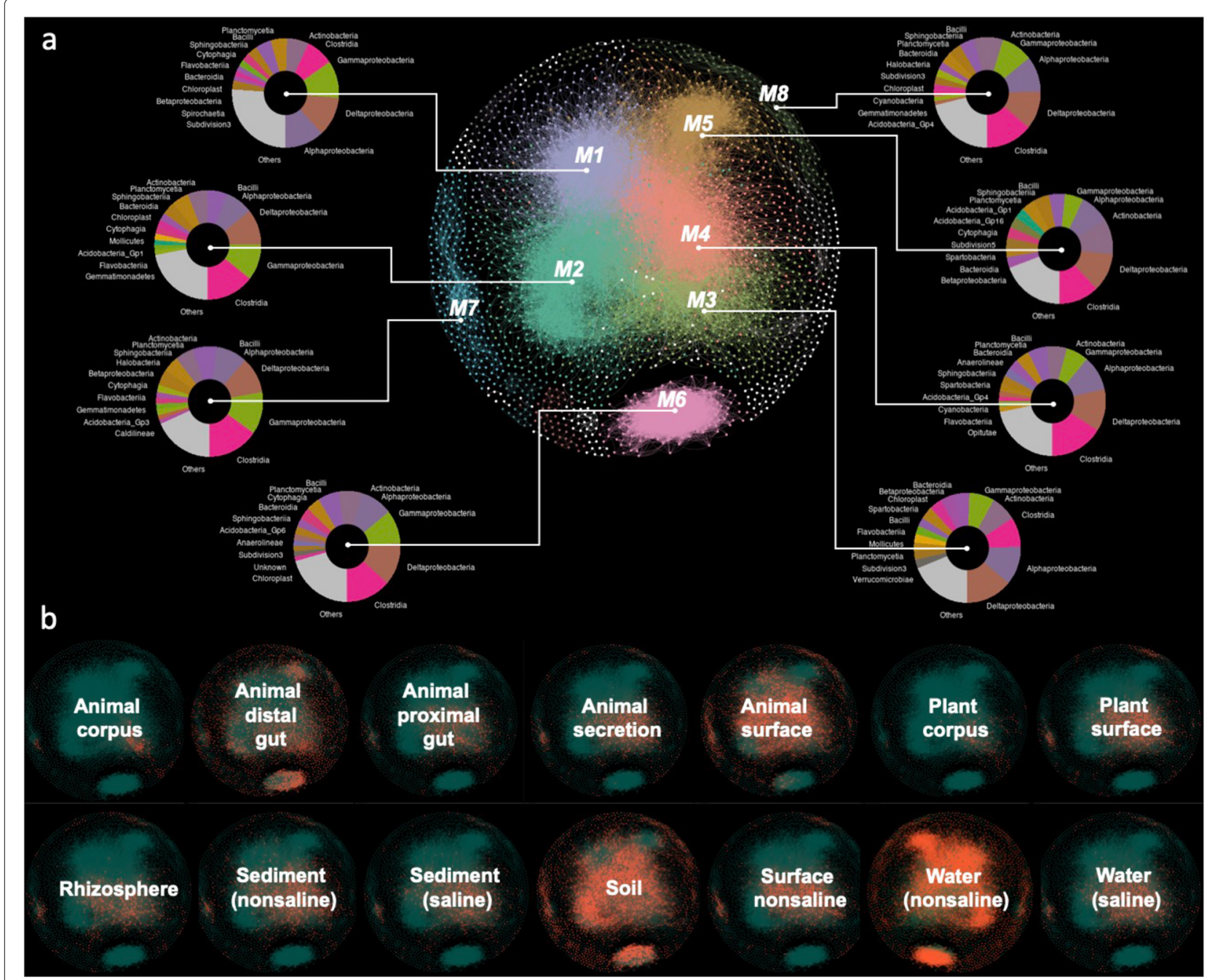

Fig. 1 Earth microbial co-occurrence network. a Layout and taxonomic profiles of eight domain modules in the Earth microbial co-occurrence network. Modules (M1-M8) are displayed in different colors. b The distribution of vertices from 14 environments in the network where orange indicates the vertices from corresponding environments

this greater module (Fig. S3). All 8 modules were comprised of different taxonomic profiles and were dominated by Clostridia, Alphaproteobacteria, Deltaproteobacteria, and Gammaproteobacteria (Fig. 1a). Vertices from microbiomes of soils, non-saline waters, animal distal guts, and animal surfaces were present in all 8 modules (Fig. 1b; Fig. S4a), and overrepresented in different modules (Fig. S4b). However, vertices from microbiomes of animal corpus were mostly restricted to and overrepresented than random frequency in M3 (3.1\%), while vertices from plant corpus comprised a major portion of and was overrepresented than random frequency in M3 (4.6\%) and M4 (2.3\%; Fig. S4a-b).

\section{Phylogeny of co-occurrence network}

With regard to phylogeny, a non-random edge distribution across taxa was observed, with most co-occurrence relationships derived from Alphaproteobacteria, Clostridia, and Deltaproteobacteria (Fig. 2a) classes. Most of the combinations between dominant classes are overrepresented than random frequency (Fig. 2b). However, only certain combinations between rare classes, such as Flavobacteriia and Gemmatinonadetes, Bacteroidia and Anaeroblineae, and Gemmatinonadetes and Bacteroidia, are overrepresented than random frequency. For within taxa co-occurrence, only co-occurrence within Deltaproteobacteria, Planctomycetia, Anaerolineae, and Acidobacteria Gp2 classes were overrepresented than random frequency. Given that the subnetworks for different environments display different co-occurrence patterns, certain co-occurrence relationships were only overrepresented than random frequency in specific environments (Fig. 2c). 


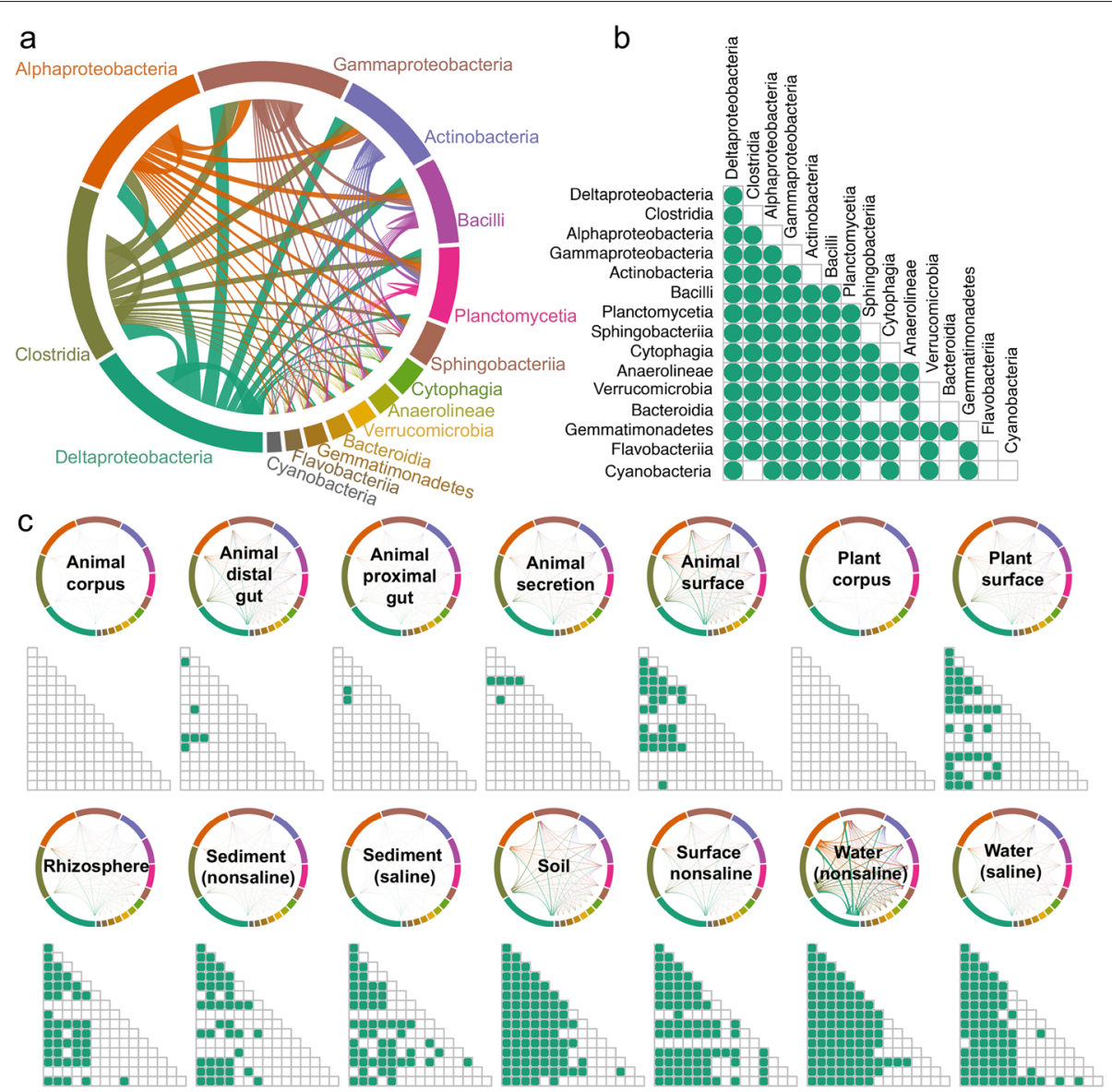

Fig. 2 Microbial co-occurrence patterns across dominant taxa. a The profiles of co-occurrence links among dominant taxa; note that connections are colored by the most dominant taxon. b Overrepresentation of co-occurrence links among taxa. The dot indicates significant overrepresentation $(P<0.05)$ between corresponding taxon pairs. c The significant overrepresentation of co-occurrence links among taxa in subnetworks for 14 environments

\section{Topological properties}

To avoid biases introduced by sample number and ESV number, we inferred 12 subnetworks for each environment with datasets trimmed into uniform size (see the "Materials and methods" section). The topological properties were highly variable between the 12 environmental subnetworks (Fig. 3). Although the datasets for 12 environments were trimmed into the same number, the edge numbers of the subnetwork of animal distal gut (4574) was 13 times larger than the subnetwork of non-saline surface (350). The diameter values ranged from 4 to 6 but were not correlated with edge numbers. The clustering coefficient values of subnetworks for animal proximal gut $(0.22)$ and saline sediment $(0.22)$ were higher than of subnetworks for other environments. The average separation $(0.30)$ and modularity (2.7) were the highest for the subnetwork of non-saline surface. Average betweenness centrality values of subnetworks of animal distal gut (212.6) and soil (206.0) were greater than those of other environments.

\section{Generalist and specialist edges}

The proportion of generalist edges, which were present in more than one subnetwork, ranged from 34.3 to $57.0 \%$ of the edges in corresponding subnetworks (Fig. 4a). Generalist edges accounted for less than $50 \%$ of edges in most subnetworks, except in non-saline water, animal secretion, and the surfaces of plants and animals. The environmental localization of generalist edges was assessed using omission scores (OS, see the "Materials and methods" section). Only $3.4 \%$ of generalist edges were identified as local edges (Data file S2).

Specialist edges, which are present in a single subnetwork, could link environment specific vertex pairs present in environment specific subnetworks or link general vertex pairs present in at least two subnetworks. The proportion of specialist edges linking specific vertex pairs accounted for $54.5 \%$ of edges in the animal proximal gut subnetwork and $52.4 \%$ of edges in the rhizosphere subnetwork, but only accounted for $15.6 \%$ of the edges in the 

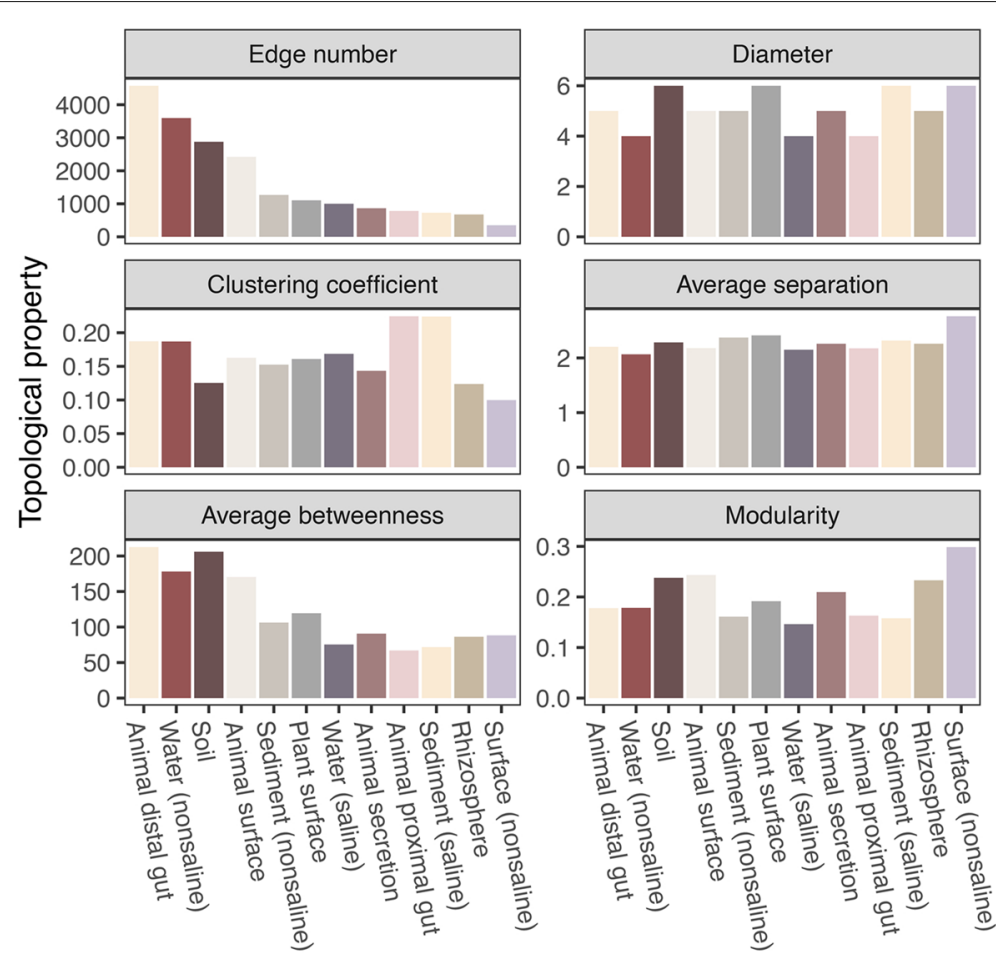

Fig. 3 Network topology of subnetworks inferred from trimmed microbiome abundance datasets of 12 environments. The microbiome abundance datasets were trimmed into 400 top-abundant ESVs and random selected 360 samples

animal secretion subnetwork. The proportion of specialist edges linking generalist vertex pairs ranged from 9.6 to $29.8 \%$ of edge numbers in corresponding subnetworks; most were greater than $20 \%$ except in the animal proximal gut $(9.6 \%)$, rhizosphere $(13.3 \%)$ and saline water $(19.1 \%)$ subnetworks. The proportions of generalist edges were negatively correlated with the proportions of specialist edges linking specific vertex pairs $(\rho=-0.87, P<0.001)$, but were not correlated with the proportion of specialist edges linking generalist vertex pairs $(\rho=0.11, P<0.72)$ (Fig. S5). Moreover, the proportions of those three edge types were not related to edge numbers in subnetworks $(P>0.10)$ (Fig. S6).

The profiles of the 50 most abundant associated vertices were different for the three edge groups (Fig. 4b). For example, Sphingobacterium was enriched in vertices associated with generalist edges, in which the most abundant edges were Sphingobacterium-Spartobacteria, Sphingobacterium-Legionella, and SphingobacteriumSolirubrobacter (Data file S2). Microgenomates was enriched in vertices associated with specialist edges linking generalist vertices, in which the most abundant co-occurrence relationships were between Microgenomates and Armatimonates. The taxa profiles of vertices associated with those three edge groups varied with environments (Fig. S7-S9).
Based on edge overlap among the subnetworks inferred from trimmed microbial community data, the 12 environments were clustered into two groups (Fig. 4c). One group consisted of the subnetworks of soil, non-saline water, animal surface, and animal distal gut (group I); the other cluster consisted of the subnetworks for rhizosphere, plant surface, secretion and proximal gut of animal, saline water and sediment, and non-saline sediment and surface (group II). Those two groups were mainly linked through the surface microbiomes of plants and animals.

\section{Network hubs}

To correct for biases of sample or taxa number, we identified the ten hubs with the highest degree from each subnetwork inferred from 12 trimmed datasets with the same sample and taxa number. A total of 120 hubs belonged to 60 ESVs (Fig. 5a), which were mainly from phyla Clostridia, Deltaproteobacteria, Alphaproteobacteria, Actinobacteria, and Gammaproteobacteria (Fig. 5b). Based on hub presentation, 12 subnetworks were clustered into two groups, which were consist with the two groups clustered based on edge overlap as described above. Acidobacteria Gp2 and Nisaea were identified as hubs in most of subnetworks. Latescibacteria was identified as hubs in all the subnetworks of soil, non-saline 

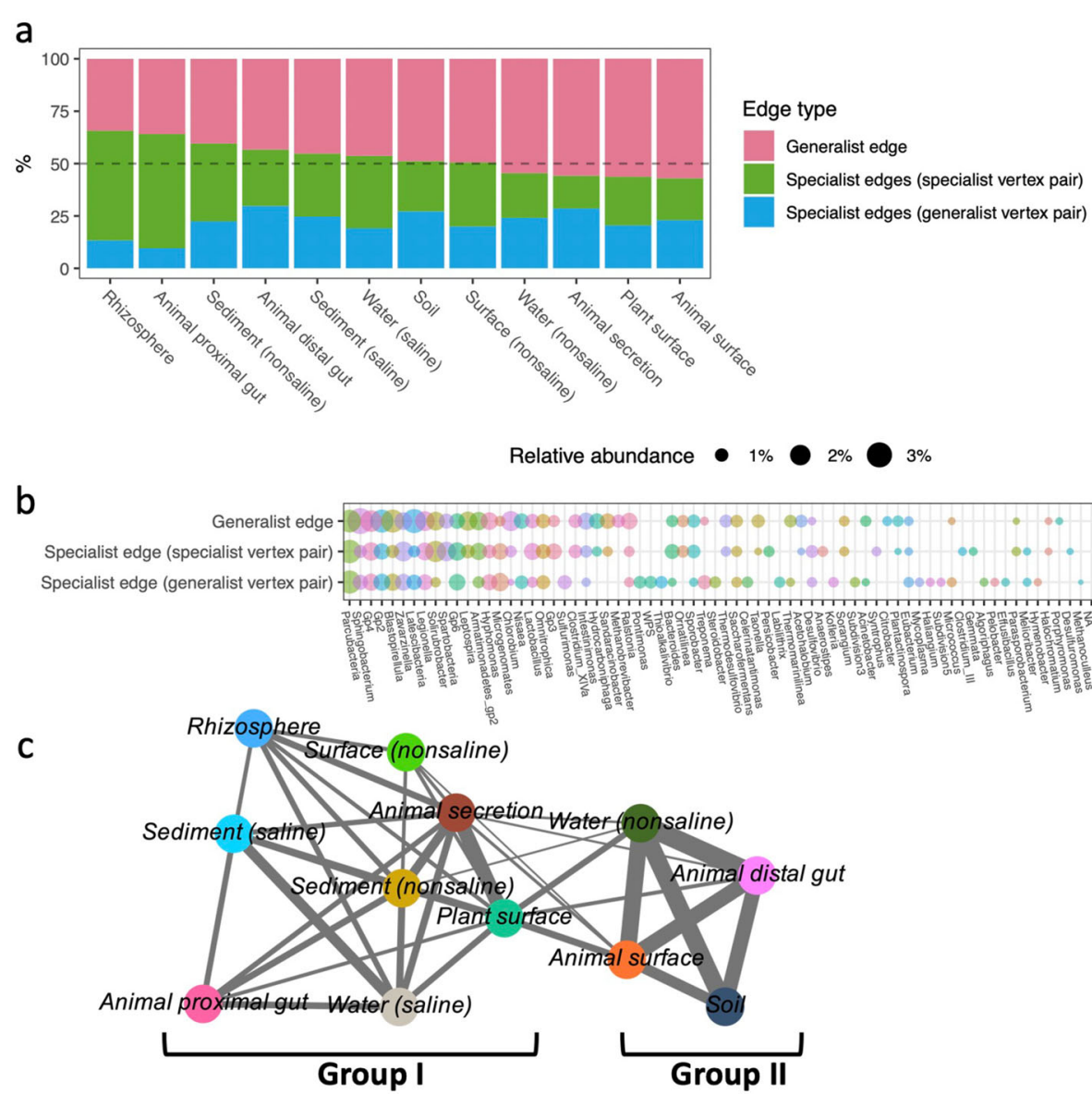

Fig. 4 Generalist and specialist edges in subnetworks inferred from trimmed microbiome abundance datasets of 12 environments. a Proportions of generalist edges, specialist edges linking specialist vertex pairs, and specialist edges linking generalist vertex pairs in 12 subnetworks. b Taxa profiles of vertices associating with generalist edges, specialist edges linking specialist vertex pairs, and specialist edges linking generalist vertex pairs. c Interconnection relationships among 12 environments based on similarity of co-occurrence relationships inferred from a Jaccard distance matrix

water, animal surface, and animal distal gut (group I). Treponema, Micrococcus, and Methanobrevibacter were identified as hubs in four of the subnetworks for rhizosphere, plant surface, secretion and proximal gut of animal, saline water and sediment, and non-saline sediment and surface (group II). Thirty-seven hubs were identified as specialist hubs, which were identified as hubs in only one subnetwork (Fig. 5a), such as in the subnetworks for soil (5), saline sediment (5), and rhizosphere (5).

\section{Negative co-occurrence links}

The proportion of negative edges ranged from 1.9 to $48.9 \%$ in the 12 subnetworks inferred from trimmed datasets (Fig. 6). Most of subnetworks consisted of more than 10\% negative edges, except in subnetworks for soil $(1.9 \%)$ and non-saline water (7.5\%). The proportion of negative edges ranged from 10.1 to $20.1 \%$ in the subnetworks for animal associated microbiomes (animal surface, secretion, and distal and proximal gut) and ranged from 27.1 to $30.8 \%$ in the subnetworks for plant-associated microbiomes (rhizosphere and plant surface). The proportion of negative edges ranged from 32.8 to $39.7 \%$ in the subnetworks for sediments and reached $48.9 \%$ in the subnetwork for nonsaline surface. Vertices linked with negative edges were dominated by phyla Alphaproteobacteria, Actinobacteria, Clostridia, Deltaproteobacteria, and Gammaproteobacteria, but the taxa profiles of negative edge-linked vertices varied with environments (Fig. 6). A substantial proportion of negative edges were linked with Acidobacteria in the subnetworks of soil, saline sediment, and animal proximal gut, with Spirochaetia in the subnetworks of saline and non-saline water, and with Sphingobacteria in the subnetworks of surface of plant, animal, and nonsaline environments. However, most negative edges were environmental specialists at genus level, except for the negative co-occurrence relationships between Spartobacteria and Acidobacteria Gp10, between Legionella and 


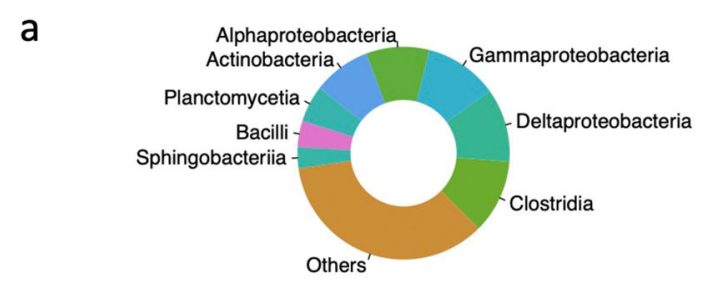

b

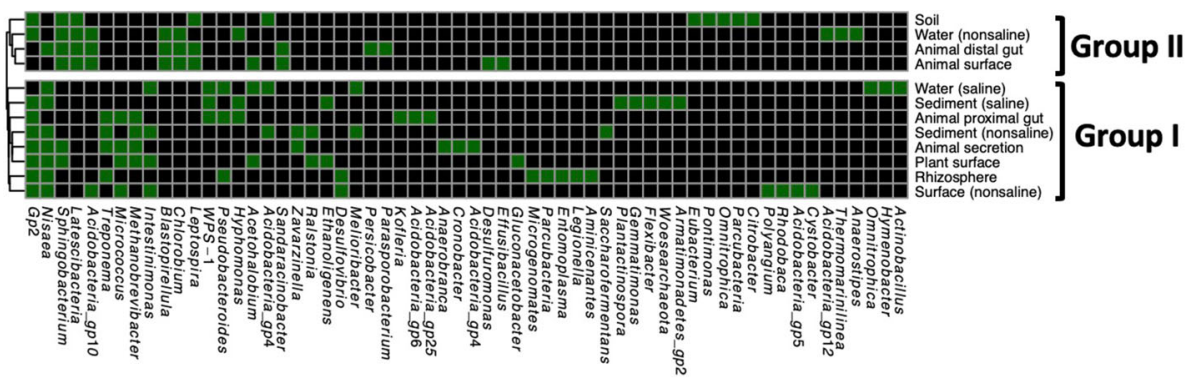

Fig. 5 Taxonomic profiles of hub insubnetworks inferred from trimmed microbiome abundance datasets of 12 environments. a The class proportion of 120 hubs in 12 subnetworks. b The genus profiles of 10 hubs in each subnetwork. The subnetworks for the 12 environments were clustered based on the taxonomic profiles of hubs in the subnetworks

Plantactinospora, and between Acidobacteria Gp6 and Acidobacteria Gp10 (Data file S3).

\section{Discussion}

We generated this global microbial co-occurrence network by taking advantage of the Earth Microbiome Project (EMP) datasets. The global microbial cooccurrence network is scale-free as has been found in other real-world networks such as the world wide web [16], social relationships [17], scientific citations [18], and interactomes of genes [19] and proteins [20]. This scalefree feature implies that a few highly connected hub species coexist with a large number of species that have a small number of links [21] and also implies an ultrasmall world network [22], which acknowledges the critical impact of microbial interaction relationships on microbial community assembly processes. Due to the nature of small world networks, impacts affecting one taxon can potentially be delivered to any other member in a microbial community via a few intermediate vertices.

The modularity of this microbial co-occurrence network varied with the environment. Previous studies have shown the existence of environmentally driven modules, such as water depth [23] or soil properties [24]. Moreover, given that modules in microbial co-occurrence networks may represent different niches [2], the present patterns of environments in modules may also indicate a similarity of microbial co-occurrence patterns in different environments. For example, similar module distributions were found for saline water and saline sediment, plant surface and rhizosphere, and the corpus of plants and animals in the current global microbial co-occurrence network.

Different profiles of topological features along the subnetworks of various environments suggest unique microbial co-occurrence patterns in different environments. Large-edge numbers in the animal distal gut subnetwork suggests a high density of interactions in that environment. Higher clustering coefficients in the subnetworks of animal proximal gut and saline sediment might be indicative of cross-feeding relationships, which suggests a richness of degradation pathways, niche filtering, or environmental heterogeneity in such environments. High average separation and modularity in the non-saline surface subnetwork suggests complex ecological processes or environment gradients in non-saline surface. Betweenness centrality measures the centrality of a vertex in a network based on shortest paths. In other words, a vertex with a higher betweenness centrality score would serve as a bridge from one part of a graph to another. The high betweenness centrality indicates that more microbes bridged connections between modules in the subnetworks of animal distal gut and soil.

Given that more than half of the edges were identified as specialist edges in most of the subnetworks, the contribution of specialist edges to co-occurrence patterns was higher than generalist edges. The primary proportion of specialist edges indicates that different environments harbored various specific microbial co-occurrence relationships. Besides specialist edges linking specialist vertices, each environment has its own specialist edges 


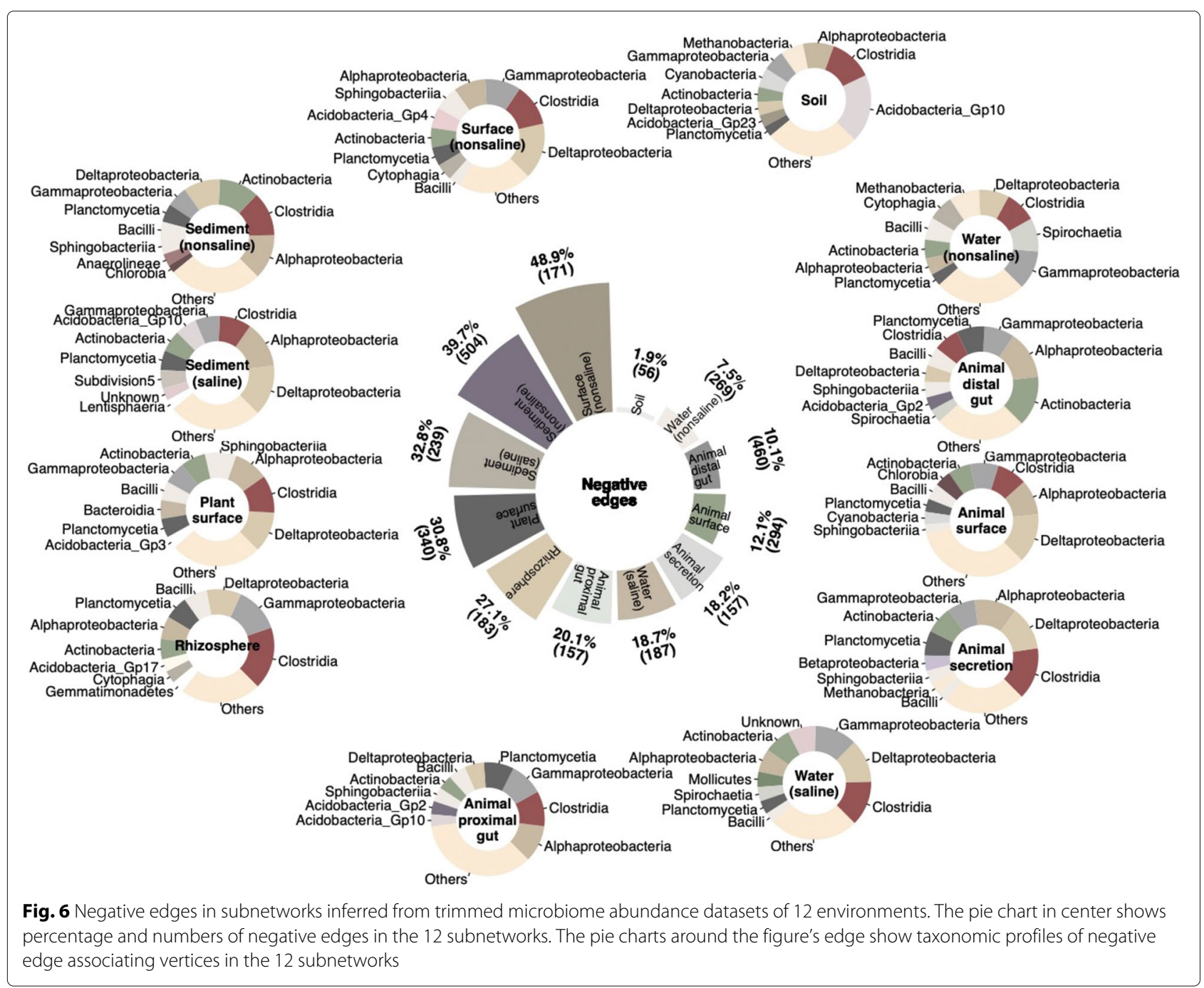

linking taxa pairs that presented in other environments as well. Based on our results, we speculate that microbial co-ocurrences could be an important aspect of describing microbial communities from different environments. Accordingly, microbial co-occurrence patterns provide a new perspective for understanding microbial community assemblages besides taxon composition in microbiomes. Although the previous finding observed major compositional distinction among the microbiomes in soil, nonsaline water, animal surface, and animal distal gut, edge overlap among subnetworks indicates a similarity of the microbial co-occurrence patterns among these environments. This cluster of two groups was in line with the subnetwork groups clustered by hub presence as well. The position of plant and animal surface microbiomes might indicate the role of these microbiomes in bridging other microbiomes. However, it is still impossible to validate the existence of inferred edges in different environments at community scale due to the high proportion of uncultured taxa in environmental microbiomes [25] and biases of primers, DNA extraction, and PCR reaction.

Different taxon profiles between generalist edges and specialist edges linking generalist vertices suggest that generalist taxa could display different co-occurrence patterns along different environments. We found that the most abundant generalist edges were all linked with Sphingobacterium, which are ubiquitous in soil [26], water [27], and animal [28] or plant-associating microbiomes [29]. However, ubiquitous existence cannot guarantee formation of generalist edges since substantial specialist edges linked generalist vertex pairs. For example, Microgenomates and Armatimonadetes are co-present in 11 environments, but only formed edges in the animal distal gut subnetwork.

The importance of hub species is intuitive because they are potentially associated with a high number of 
other species. The high degree of Acidobacteria Gp2 and Nisaea in most of the environments may be explained by their high prevalence and possibly by their generalist lifestyles [30]. Given that Acidobacteria Gp and Nisaea acquired edges with various taxonomic profiles in different environments, those genera may have the potential to synchronize ecological processes over broad ecosystems. Latescibacteria, present in specialist hubs in group I environment subnetworks, is from an uncultured candidate phylum. Its genomic segments recovered from metagenome analyses demonstrate that it is prevalent in a wide range of habitats, but that various Latescibacteria strains prefer specific habitats and have different ecological functions [31]. Latescibacteria could play an important role in the production of cellulosomes in anaerobic habitats, such as in animal guts and sediments, and in polysaccharide degradation in soils [31]. These ecological functions might make Latescibacteria a specialist hub in the subnetworks of group I environments. Treponema, the hub in the subnetwork of animal distal gut, is a characteristic symbiont in human gut microbiomes [32]. Micrococcus, the hub in the subnetworks of plant surface and non-saline surface, plays critical roles in biofilm formation [33]. Methanobrevibacter, the hub in subnetworks of animal distal gut, positively correlated with 20 hydrogen-producing Clostridales in human gut [34]. Moreover, a high proportion of specialist hubs suggests that hubs could represent co-occurrence characteristics in various environments. We note that it is difficult to infer hub nodes correctly [2] and that it is not yet clear whether hub node status also implies a special role in the ecosystem in the sense of a keystone, though initial experiments suggest this is the case [35].

Negative edges may originate from a wide range of co-exclusion mechanisms, including direct competition, toxin production, environmental modification, and differential niche adaptation [36]. Different proportions of negative edges suggest various intensities of competition or niche differentiation in different environments. Low proportions of negative edges in soil subnetworks suggest a prevalence of collaboration or niche sharing in soil, in which heterogeneous microenvironments could reduce direct competition. A large proportion of negative edges in the soil subnetworks linked with Acidobacteria, which are ubiquitous in soil environments but are under-represented in culture studies [37]. The ecological capabilities of Acidobacteria predicted by a metagenomic approach alludes to a competitive life style in soils [37]. High proportions of negative edges in sediments and surface data suggest that competition or subniche differentiation were more prevalent in sediment and surface environments. Compared with the soil, fewer ecological niches exist in sediment and surface environments due to their relatively homogenous microenvironments. The proportion of negative edges in the subnetworks of plantassociated microbiomes was higher than in the subnetworks of animal associated microbiomes, suggesting that competition or niche differentiation was more prevalent in plant-associated microbiomes.

\section{Conclusions}

In summary, the present study provides an overview of global microbial co-occurrence patterns. With this study, we have shown the interconnection pattern across environments in the Earth microbial co-occurrence network. Moreover, we suggest that microbial co-occurrence pattern is a critical aspect of microbial community characteristic that can be used in conjunction with microbial taxon compositional profiles. Given the increasing recognition of the value of communal microbial biodiversity monitoring and the current global advance in sequencing techniques, future sequencing efforts will likely increase the accuracy of the global microbial co-occurrence network presented in this study. Given that most microbial co-occurrence relationships lacked experimental validation, a greater effort is needed to mine uncultured microbial species for validating predicted microbial cooccurrence relationships with co-culture experiments. In addition, the EMP datasets currently focus on bacterial and archaeal communities, but other life forms on Earth (for example plants, animals, and fungi) are also essential in the microbial interactome due to their influences on microbial environments. Future studies filling the gaps for microbial eukaryotes within the EMP framework will untangle global microbial co-occurrence patterns comprehensively.

\section{Materials and methods}

\section{Abundance table from the EMP}

The microbial abundance table used in the present study was the 90-bp Deblur BIOM table from the EMP database [15]. This table was based on the sequence data from the EMP databased after filtering errors and trimming to $90 \mathrm{bp}$ (the length of the shortest sequencing run) using Deblur in Qiime2 [38]. The EMP employed a unified standard workflow for soil collection, metadata curation, DNA extraction, sequencing, and sequence preprocessing, to avoid known issues in combining multiple amplicons across diverse environments on Earth. The abundance table was filtered to keep tag sequences with at least 25 reads total over all samples. We then extracted 14 count matrices for 14 environmental categories at level 3 of the EMP ontology (Table S1) from the 90bp Deblur BIOM table [15]. We filtered the ESVs with relative abundance less than $0.001 \%$ and presenting in less than $10 \%$ of samples in corresponding count matrices of environments. All the analyses were done using R 3.6.0 [39]. 


\section{Network inference}

Microbial taxon-taxon co-occurrence networks were constructed as described by Lima-Mendez et al. [13] by selecting Spearman correlation and Bray-Curtis dissimilarity measures. Briefly, to compute $P$ values, we generated permutation and bootstrap distributions (1000 iterations each) by shuffling taxon abundances and resampling from samples with replacements, respectively. The $P$ value was then obtained as the probability of the null value under a Gaussian curve fitted to the mean and standard deviation of the bootstrap distribution. Permutations computed for the Spearman correlation included a renormalization step to mitigate compositionality bias. Measure-specific $P$ values were merged using Brown's method [40] and multiple-testing-corrected with the Benjamini-Hochberg method [41]. Finally, edges with an adjusted $P$ value above 0.05 and a score below the thresholds determined with random matrix theory method [42] or not supported by both measures after assessment of significance were discarded. For computational efficiency, we computed 14 taxon-taxon networks separately for 14 environmental categories at level 3 of EMP ontology. Network deconvolution was employed for detecting indirect co-occurrences in those networks $(\alpha=1, \beta=0.9)$ [43]. The 14 taxon-taxon networks were then merged into a global network.

\section{Trimmed microbial community dataset}

In order to avoid the taxon or sample number biases, we trimmed each community dataset of various environments into the same taxon number and the same sample number. We kept 400 top-abundant ESVs and randomly selected 360 samples in the trimmed microbial community matrices. Due to smaller size than trimmed matrices, the microbiomes in plant and animal corpus were not involved in inferring subnetworks with trimmed dataset.

\section{Influence of environment on co-occurrence pattern}

The impact of environmental categories on the Spearman correlation of each edge in the network was assessed through dividing the absolute omission score (OS) (Spearman correlation without the environmental categories) by the absolute original Spearman score. To account for group size, the OS was computed repeatedly for random, same-sized sample sets. Nonparametric $P$ values were calculated as the number of times random OSs were smaller than the sample group OS, divided by the number of random OS (1000 for each taxon pair). Edges were classified as region-specific when the ratio of OS and absolute original score was below 1 , and the multiple-testing-corrected $P$ values (Benjamini-Hochberg) were below 0.05 .

\section{Overrepresentation analysis}

Statistics were done using stats package in $\mathrm{R}$ 3.6.0 [39]. Taxon-taxon counts at high taxonomic ranks were assessed for overrepresentation significance using the hypergeometric distribution implemented by stats :: phyper. Mutual exclusion versus co-presence analysis was performed using the binomial distribution implemented by stats :: pbinom, with background probability estimated by the frequency of edges in the network. In all tests, $P$ values were adjusted for multiple testing according to Benjamini, Hochberg, and Yekutieli (BY); adjustments were made using the stats :: p.adjust.

\section{Literature-based evaluation of predicted co-occurrence relationships}

We counted known species pairs of co-occurrence relationships and filtered the co-occurrence pairs presenting less than three times in the network. Then, we built a list of 812 pairs of species co-occurrence relationships with significant overrepresentation $(P<0.05)$. We screened the literature retrieved from Web of Science by querying two species names of a specific co-occurrence relationship and confirm the relationships reported in literature. The protocol to screen the literature was the following: (i) we screened returned literature for direct observed relationship, such as competition or mutualism; (ii) if no direct relationships, we screened returned literature for co-occurrence in the same samples; (iii) if no cooccurrence, we checked if the two species belonged to the same taxon; (iv) otherwise, the edges was labeled as unpublished relationships.

\section{Topological features}

Topological features were estimated with igraph package (v1.4.1) [44] in R 3.6.0 [39]. Edge number was determined using the ecount function, and diameter was determined using the diameter function. Clustering coefficient was estimated with the transitivity function and average separation was estimated with the mean_distance function. Mean betweenness centrality was calculated using the centr_betw function. Modularity was estimated with the modularity function based on the fast greedy clustering algorithm.

\section{Generalist and specialist edges}

Edges present in only one subnetwork were specialist edges, which were further clustered into two groups: a specialist edge linking a specialist vertex pair or the same linking a generalist vertex pair. A specialist edge linking specialist vertex pair represents the contribution of environmental-specific taxa in specialist edges, while a specialist edge linking generalist vertex pair represents the potential contribution of the environment in enriching specific microbial interactions. The 50 top-abundant ESVs for each edge type were counted for taxon profile comparison. An environment similarity network was inferred with a Jaccard distance matrix based on edge overlap 
among subnetworks inferred with the trimmed dataset. The spearman correlation between different edge types and between edge number and edge types was calculated using the cor.test function.

\section{Hub identification}

We identified ten hubs at the top-degree from each subnetwork inferred from the 12 trimmed datasets. The taxon profiles of hubs in different subnetworks were identified at genus level with the 90-bp Deblur BIOM table [15].

\section{Negative edges}

We counted the number and percentage of negative edges in the subnetworks inferred from the 12 trimmed datasets. The taxon profiles of negative edges in the subnetworks of various environments were estimated with the class groups of both vertices.

\section{Supplementary information}

Supplementary information accompanies this paper at https://doi.org/10.1186/s40168-020-00857-2.

\section{Data file S1: Data file S1. Dataset of literature validation records. Data file S2: Data file S2. Dataset of edge frequency. \\ Data file S3: Data file S3. Dataset of negative edges. \\ Supplementary materials:}

Fig. S1. The degree distribution of Earth microbial co-occurrence network. Fig. S2. The relative abundance of vertices in 8 modules of the Earth microbial co-occurrence network. Fig. S3. The co-occurrence across 8 modules of the Earth microbial co-occurrence network. Fig. S4. The distribution of vertices from subnetworks for 14 environmental types among 8 dominant modules. Fig. S5. Relationships between proportions of generalist edge, specialist edge linking generalist vertex pair, and specialist edge linking specialist vertex pair. Fig. S6. Relationship between edge number and generalist edge, specialist edge linking generalist vertex pair, and specialist edge linking specialist vertex pair. Fig. S7. Taxon profiles of generalist edge linked vertices in subnetworks of 12 environments inferred from trimmed datasets. Fig. S8. Taxon profiles of specialist edge linking generalist vertex pair linked vertices in subnetworks of 12 environments inferred from trimmed datasets. Fig. S9. Taxon profiles of specialist edge linking specialist vertex pair linked vertices in subnetworks of 12 environments inferred from trimmed datasets.

\section{Acknowledgements}

Not applicable.

\section{Authors' contributions}

$B M, J G, K F$, and JX designed the study. BM, ES, LR, CC, and JX wrote the manuscript. YW, SY, SL, and ES performed the literature validation, JA, RK, and JK parse the EMP dataset. BM, KF, and LR performed the network analysis. BM performed the figure visualization. The authors read and approved the final manuscript.

\section{Funding}

This research was financially supported by the National Natural Science Foundation of China (41721001, 41991334), the Zhejiang Provincial Natural Science Foundation of China (LD19D060001), the 111 Project (B17039), and the Fundamental Research Funds for the Central Universities. Partial support was provided through the Microbiomes in Transition (MinT) Initiative under the Laboratory Directed Research and Development Program at PNNL (PNNL is a multiprogram national laboratory operated for DOE by Battelle Memorial Institute under contract DE-AC06-76RL01830).

\section{Availability of data and material}

The EMP dataset are available at ftp://ftp.microbio.me/emp/release1. The R script used in this study and network files are available at http://www.github. $\mathrm{com} / \mathrm{microbma/earthnetwork/.}$

Ethics approval and consent to participate

Not applicable.

Consent for publication

Not applicable.

\section{Competing interests}

The authors declare that they have no competing interests.

\section{Author details}

${ }^{1}$ Institute of Soil and Water Resources and Environmental Science, College of Environmental and Resource Sciences, Zhejiang University, Hangzhou, 310058, China. ${ }^{2}$ Zhejiang Provincial Key Laboratory of Agricultural Resources and Environment, Hangzhou, 310058, China. ${ }^{3}$ Department of Pediatrics and Scripps Institution of Oceanography, University of California San Diego, La Jolla, CA USA. ${ }^{4}$ Department of Microbiology and Immunology, Rega Institute, KU Leuven, Campus Gasthuisberg, Leuven, Belgium. ${ }^{5}$ Departments of Pediatrics, Computer Science and Engineering, and BioEngineering, University of California San Diego, La Jolla, CA USA. ${ }^{6}$ Biological Sciences Division, Earth and Biological Sciences Directorate, Pacific Northwest National Laboratory, Richland, WA 99352, USA. ${ }^{7}$ Graduate Program in Biophysical Sciences, The University of Chicago, Chicago IL, 60637, USA.

Received: 5 February 2020 Accepted: 7 May 2020

Published online: 04 June 2020

\section{References}

1. Faust K, Raes J. Microbial interactions: from networks to models. Nat Rev Microbiol. 2012;10:538-50.

2. Röttjers $L$, Faust K. From hairballs to hypotheses-biological insights from microbial networks. FEMS Microbiol Rev. 2018;42:761-80.

3. Stenseth NC, Smith JM. Coevolution in ecosystems: Red Queen evolution or stasis? Evolution. 1984;38:870-80.

4. Morris JJ, Lenski RE, Zinser ER. The Black Queen hypothesis: evolution of dependencies through adaptive gene loss. mBio. 2012;3:e00036-12.

5. Decaestecker E, Gaba S, Raeymaekers JA, Stoks R, Van Kerckhoven L, Ebert D, et al. Host-parasite 'Red Queen' dynamics archived in pond sediment. Nature. 2007;450:870

6. Fisher RM, Henry LM, Cornwallis CK, Kiers ET, West SA. The evolution of host-symbiont dependence. Nat Commun. 2017;8:15973.

7. Cordero OX, Polz MF. Explaining microbial genomic diversity in light of evolutionary ecology. Nat Rev Microbiol. 2014;12:263-73.

8. Hughes AR, Inouye BD, Johnson MT, Underwood N, Vellend M. Ecological consequences of genetic diversity. Ecol Lett. 2008;11:609-23.

9. Paramsothy S, Kamm MA, Kaakoush NO, Walsh AJ, van den Bogaerde J, Samuel D, et al. Multidonor intensive faecal microbiota transplantation for active ulcerative colitis: a randomised placebo-controlled trial. Lancet. 2017;389:1218-28.

10. O'Callaghan M. Microbial inoculation of seed for improved crop performance: issues and opportunities. Appl Microbiol Biotechnol. 2016;100:5729-46.

11. Li SS, Zhu A, Benes V, Costea PI, Hercog R, Hildebrand F, et al. Durable coexistence of donor and recipient strains after fecal microbiota transplantation. Science. 2016;352:586-9.

12. Berry $\mathrm{D}$, Widder S. Deciphering microbial interactions and detecting keystone specieswith co-occurrence networks. Front Microbiol. 2014;5:219.

13. Lima-Mendez G, Faust K, Henry N, Decelle J, Colin S, Carcillo F, et al. Determinants of community structure in the global plankton interactome. Science. 2015;348:1262073.

14. Chaffron S, Rehrauer H, Pernthaler J, von Mering C. A global network of coexisting microbes from environmental and whole-genome sequence data. Genome Res. 2010;20:947-59.

15. Thompson LR, Sanders JG, McDonald D, Amir A, Ladau J, Locey KJ, et al. A communal catalogue reveals Earth's multiscale microbial diversity. Nature. 2017;551:457-63.

16. Albert $\mathrm{R}$, Jeong $\mathrm{H}$, Barabási A-L. Internet Diameter of the world-wide web. Nature. 1999;401:130. 
17. Liljeros F, Edling CR, Amaral LAN, Stanley HE, Aberg Y. The web of human sexual contacts. Nature. 2001;411:907.

18. Wang D, Barabási AL. Quantifying long-term scientific impact. Science. 2013;342:127-32.

19. Ma B, Zhao K, LvX, Su W, Dai Z, Gilbert JA, et al. Genetic correlation network prediction of forest soil microbial functional organization. ISME J. 2018;12:2492-505.

20. Eisenberg E, Levanon EY. Preferential attachment in the protein network evolution. Phys Rev Lett. 2003;91:138701

21. Barabási AL. Scale-free networks: a decade and beyond. Science. 2009;325:412-3

22. Amaral LAN, Scala A, Barthelemy M, Stanley HE. Classes of small-world networks. Proc Natl Acad Sci USA. 2000;97:11149-52.

23. Cram JA, Xia LC, Needham DM Sachdeva R, Sun F, Fuhrman JA. Cross-depth analysis of marine bacterial networks suggests downward propagation of temporal changes. ISME J. 2015;9:2573.

24. Jiang Y, Sun B, Li H, Liu M, Chen L, Zhou S. Aggregate-related changes in network patterns of nematodes and ammonia oxidizers in an acidic soil. Soil Biol Biochem. 2015;88:101-9.

25. Lok C. Mining the microbial dark matter. Nat News. 2015;522:270.

26. Zhou X-K, Li Q-Q, Mo M-H, Zhang Y-G, Dong L-M, Xiao M, et al. Sphingobacterium tabacisoli sp. nov., isolated from a tobacco field soil sample. Int J Syst Evol Microbiol. 2017;67:4808-13.

27. Albert RA, Waas NE, Pavlons SC, Pearson JL, Ketelboeter $L$, Rosselló-Móra R, et al. Sphingobacterium psychroaquaticum sp. nov., a psychrophilic bacterium isolated from Lake Michigan water. Int J Syst Evol Microbiol. 2013;63:952-8.

28. Zhou J, Huang $H$, Meng K, Shi P, Wang Y, Luo H, et al. Molecular and biochemical characterization of a novel xylanase from the symbiotic Sphingobacterium sp. TN19. Appl Microbiol Biotechnol. 2009;85:323-33.

29. Kömpfer P, Busse H-J, Kleinhagauer T, Mclnroy JA, Glaeser SP. Sphingobacterium zeae sp. nov., an endophyte of maize. Int J Syst Evol Microbiol. 2016;66:2643-9

30. Freilich R, Arhar T, Abrams JL, Gestwicki JE. Protein-protein interactions in the molecular chaperone network. Acc Chem Res. 2018;51:940-9.

31. Farag IF, Youssef $\mathrm{NH}$, Elshahed MS. Global distribution patterns and pangenomic diversity of the candidate phylum "Latescibacteria" (WS3) Löffler FE, editor. Appl Environ Microbiol. 2017;83:e00521.

32. Obregon-Tito AJ, Tito RY, Metcalf J, Sankaranarayanan K, Clemente JC, Ursell LK, et al. Subsistence strategies in traditional societies distinguish gut microbiomes. Nat Commun. 2015;6:6505.

33. Blakeman JT, Morales-García AL, Mukherjee J, Gori K, Hayward AS, Lant $\mathrm{NJ}$, et al. Extracellular DNA provides structural integrity to a micrococcus luteus biofilm. Langmuir ACS J Surf Colloids. 2019;35:6468-75.

34. Hansen EE, Lozupone CA, Rey FE, Wu M, Guruge JL, Narra A, et al. Pan-genome of the dominant human gut-associated archaeon, Methanobrevibacter smithii, studied in twins. Proc Natl Acad Sci USA. 2011;108:4599-606.

35. Agler MT, Ruhe J, Kroll S, Morhenn C, Kim S-T, Weigel D, et al. Microbial hub taxa link host and abiotic factors to plant microbiome variation. Waldor MK, editor. PLOS Biol. 2016;14:e1002352.

36. Faust K, Sathirapongsasuti JF, Izard J, Segata N, Gevers D, Raes J, et al. Microbial co-occurrence relationships in the human microbiome. PLoS Comput Biol. 2012;8:e1002606.

37. Kielak AM, Barreto CC, Kowalchuk GA, van Veen JA, Kuramae EE. The ecology of Acidobacteria: moving beyond genes and genomes. Front Microbiol. 2016;7:744.

38. Amir A, McDonald D, Navas-Molina JA, Kopylova E, Morton JT, Xu ZZ, et al. Deblur rapidly resolves single-nucleotide community sequence patterns. mSystems. 2017;2:e00191-16.

39. R Core Team. R: a language and environment for statistical computing. $R$ Foundation for Statistical Computing. Vienna, Austria. https://www.Rproject.org/.

40. Poole W, Gibbs DL, Shmulevich I, Bernard B, Knijnenburg TA. Combining dependent $P$-values with an empirical adaptation of Brown's method. Bioinformatics. 2016;32:i430-6.

41. Benjamini $Y$, Yekutieli $D$. The control of the false discovery rate in multiple testing under dependency. Ann Stat. 2001;29:1165-88.

42. Luo F, Zhong J, Yang Y, Scheuermann RH, Zhou J. Application of random matrix theory to biological networks. Phys Lett A. 2006;357:420-3.
43. Feizi S, Marbach D, Médard M, Kellis M. Network deconvolution as a general method to distinguish direct dependencies in networks. Nat Biotechnol. 2013;31:726-33.

44. Csardi G, Nepusz T. The igraph software package for complex network research. InterJournal Complex Syst. 2006;1695:1-9.

\section{Publisher's Note}

Springer Nature remains neutral with regard to jurisdictional claims in published maps and institutional affiliations.

\section{Ready to submit your research? Choose BMC and benefit from:}

- fast, convenient online submission

- thorough peer review by experienced researchers in your field

- rapid publication on acceptance

- support for research data, including large and complex data types

- gold Open Access which fosters wider collaboration and increased citations

- maximum visibility for your research: over $100 \mathrm{M}$ website views per yea

At $B M C$, research is always in progress.

Learn more biomedcentral.com/submission 